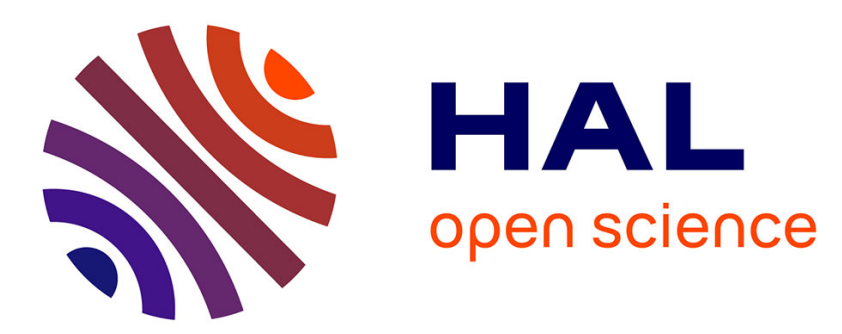

\title{
Commutations optiques ultra-rapides dans un film non-linéaire de silicium sur saphir
}

H. Chelli, A. Koster, N. Paraire, F. Pardo, Hervé Sauer, M. Carton, S. Laval

\section{To cite this version:}

H. Chelli, A. Koster, N. Paraire, F. Pardo, Hervé Sauer, et al.. Commutations optiques ultra-rapides dans un film non-linéaire de silicium sur saphir. Revue de Physique Appliquée, 1987, 22 (10), pp.12731279. 10.1051/rphysap:0198700220100127300 . jpa-00245677

\section{HAL Id: jpa-00245677 https://hal.science/jpa-00245677}

Submitted on 1 Jan 1987

HAL is a multi-disciplinary open access archive for the deposit and dissemination of scientific research documents, whether they are published or not. The documents may come from teaching and research institutions in France or abroad, or from public or private research centers.
L'archive ouverte pluridisciplinaire HAL, est destinée au dépôt et à la diffusion de documents scientifiques de niveau recherche, publiés ou non, émanant des établissements d'enseignement et de recherche français ou étrangers, des laboratoires publics ou privés. 


\title{
Commutations optiques ultra-rapides dans un film non-linéaire de silicium sur saphir
}

\author{
H. Chelli, A. Koster, N. Paraire, F. Pardo, H. Sauer, M. Carton et S. Laval \\ Institut d'Electronique Fondamentale, Université Paris XI, CNRS UA 22, Bâtiment 220, 91405 Orsay Cedex, \\ France
}

(Reçu le 13 novembre 1986, accepté le 4 mars 1987)

\begin{abstract}
Résumé. - Un dispositif original de commutation entièrement optique, utilisant l'excitation de modes guidés dans un film de silicium sur saphir est présenté. Cette structure a permis l'observation de commutations rapides (quelques centaines de picosecondes). Une analyse théorique est exposée qui permet d'expliquer les observations expérimentales et de définir les conditions d'utilisation permettant d'obtenir la bistabilité.
\end{abstract}

\begin{abstract}
An intrinsic optical switching device, using the excitation of guided modes in a silicon on sapphire film is presented. This structure allows the observation of fast switching (a few hundred picoseconds). A theoretical analysis is presented which explains the experimental observations and allows to predict in which conditions bistability can be observed.
\end{abstract}

Différentes structures ont été envisagées au cours des dernières années pour obtenir des commutations tout-optiques ultra-rapides $[1,2]$. Dans chaque cas, outre la rapidité des transitions, plusieurs facteurs interviennent dans l'optimisation de ces dispositifs : énergie nécessaire à la commutation, dimensions géométriques, maîtrise des processus technologiques de réalisation... Nous présentons une structure originale de dispositifs de commutation entièrement optique qui utilise l'excitation d'un mode guidé dans un film mince de silicium sur saphir. Cette structure a permis l'observation de commutations rapides (quelques centaines de picosecondes) et bien contrastées. Dans ce qui suit nous décrivons les résultats expérimentaux, nous les analysons théoriquement en mettant en évidence l'influence des problèmes de dissipation thermique et proposons des conditions d'utilisation pour l'observation de cycles de bistabilité.

\section{Description du dispositif expérimental.}

Nous utilisons un guide non linéaire de silicium épitaxié sur du saphir et recouvert d'argent dans lequel nous étudions la propagation de modes TE. Les épaisseurs du silicium et du saphir sont respectivement de $0,7 \mu \mathrm{m}$ et $325 \mu \mathrm{m}$ (Fig. 1).

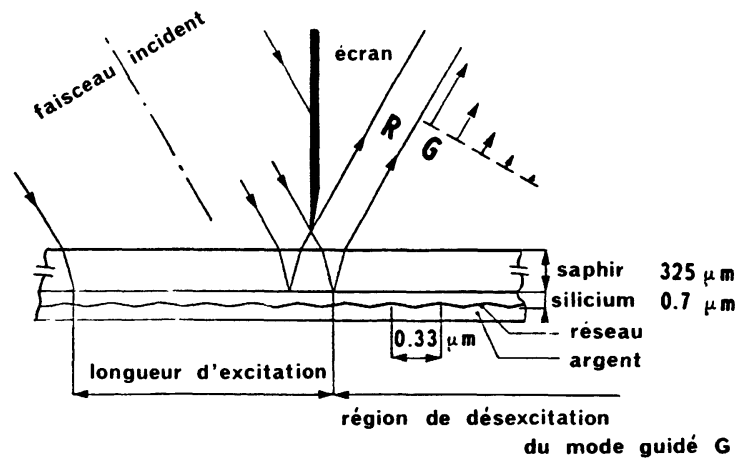

Fig. 1. - Structure du dispositif.

[Device structure.]

La longueur d'onde excitatrice $(1,064 \mu \mathrm{m})$ correspond au bord de la bande d'absorption du silicium, zone dans laquelle les non-linéarités sont importantes. Lors de la propagation des modes dans le guide, il y a réflexion totale de la lumière aux interfaces saphir-silicium et silicium-argent : on observe donc un très bon confinement de la lumière. Le dispositif est comparable à un résonateur Fabry-Pérot de très faible épaisseur $(0,7 \mu \mathrm{m})$ et possédant un coefficient de surtension très élevé; par rapport à un tel 
dispositif, il doit cependant présenter une dimension latérale minimale correspondant à la distance nécessaire à la construction des modes. Le couplage entre le guide et un faisceau incident est réalisé grâce à un réseau dont le pas $(0,3306 \mu \mathrm{m})$ a été choisi pour qu'il n'y ait qu'un ordre diffracté rayonné et pour que les différents modes TE soient commodément excités. La profondeur de modulation peut être choisie pour permettre un couplage total pour un mode particulier [3].

Lors des mesures, on envoie sur le dispositif un faisceau laser, sous une incidence $\theta$ et on observe dans la direction de la lumière réfléchie. Un écran permet de délimiter spatialement dans cette direction une zone $\mathrm{R}$ où les phénomènes observés correspondent essentiellement à ceux créés par une excitation en onde plane, et une zone $G$ où, en l'absence de lumière réfléchie, on observe l'onde de fuite associée au mode guidé. L'image sur un trou d'une partie du dispositif permet l'analyse spatiale des régions $\mathrm{R}$ et $\mathrm{G}$; cette analyse est effectuée grâce à un déplacement relatif du faisceau excitateur par rapport à l'ensemble écran-dispositif de détection (Fig. 2).

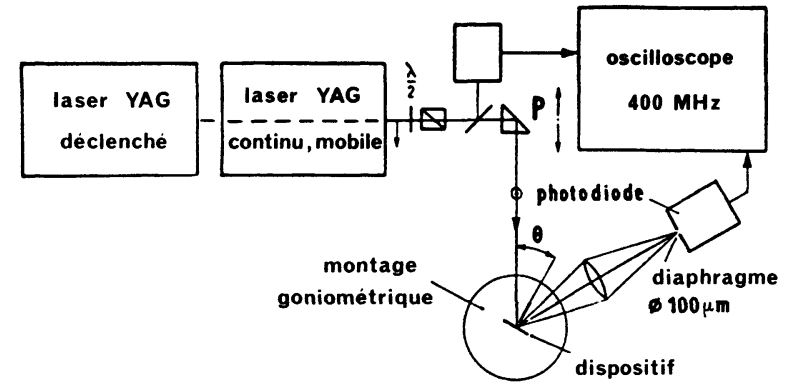

Fig. 2. - Dispositif expérimental.

[Experimental set-up.]

\section{Observation expérimentale.}

Une étude préliminaire, effectuée en utilisant un faisceau incident continu de faible puissance, permet de déterminer la position angulaire et la largeur des modes. L'étude en régime non linéaire est ensuite réalisée en utilisant un laser Nd-Yag délivrant des impulsions de $20 \mathrm{~ns}$; la divergence du faisceau est de $0,2 \mathrm{mrad}$ et son intensité peut être ajustée par un système lame demi-onde/polariseur. Sur la figure 3 nous avons reporté les observations faites sur le mode fondamental $\mathrm{TE}_{0}$, qui présente la résonance la plus aiguë, dans les régions $\mathrm{R}$ et $\mathrm{G}$, en continu, puis en impulsion pour de faibles valeurs de la puissance incidente. La résonance s'observe pour $\theta_{0}=14,79^{\circ}$, la largeur de la courbe étant $\delta \theta \approx 0,2^{\circ}$. Cette résonance aiguë permet d'observer des effets non linéaires (commutations ou cycles de bistabilité) à des puissances incidentes raisonnables pour des valeurs de l'angle d'incidence différentes.

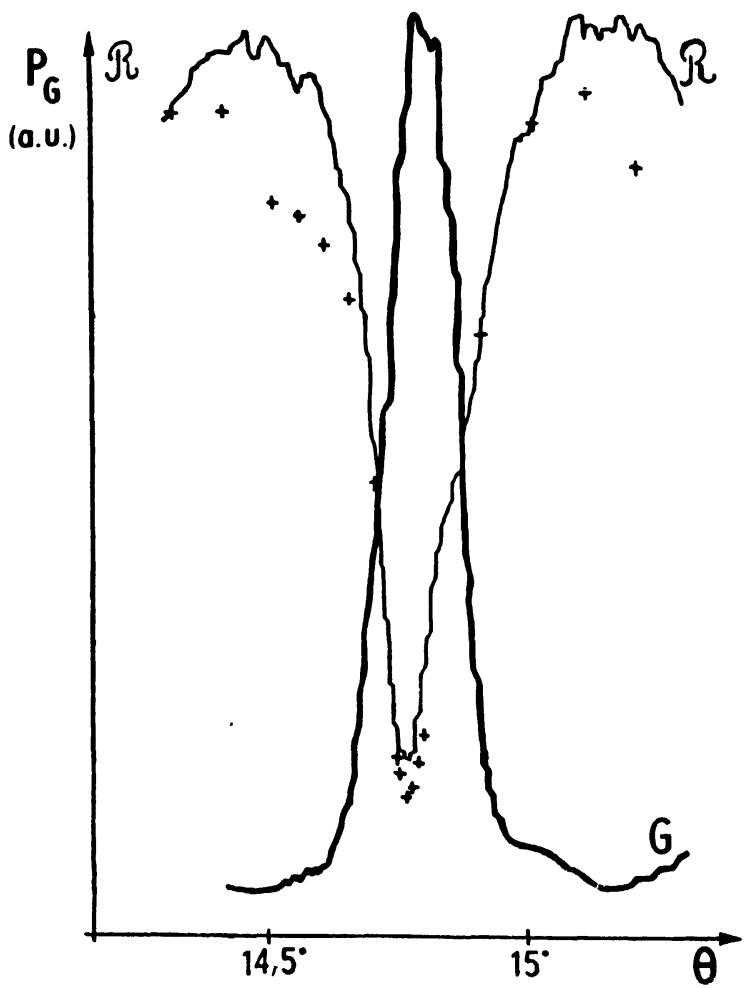

Fig. 3. - Coefficient de réflexion et intensité guidée en fonction de l'angle d'incidence pour le mode fondamental $\mathrm{TE}_{0}$. — : enregistré avec un laser Nd-Yag continu. + : mesuré avec un laser Nd-Yag déclenché fonctionnant à faible puissance.

[Reflection coefficient $(\mathcal{R})$ and guided intensity $\left(P_{\mathrm{G}}\right)$ versus the incidence angle for the $\mathrm{TE}_{0}$ fundamental mode. - : recorded with a $\mathrm{CW}$ Nd-Yag laser. + : measured with a low power Q-Switched Nd-Yag laser.]

Notons cependant que sous l'action d'un rayonnement, la non-linéarité du silicium peut avoir deux causes :

- une variation d'indice d'origine électronique $\delta n_{\mathrm{e}}$, proportionnelle à l'intensité guidée, et négative [4],

- une variation d'indice d'origine thermique $\delta n_{\mathrm{T}}$, due à l'échauffement du matériau par absorption du rayonnement, et positive.

La variation globale d'indice observée $\delta n=$ $\delta n_{\mathrm{e}}+\delta n_{\mathrm{T}}$ dépend de la compétition entre ces deux phénomènes.

La réflectivité $\mathcal{R}(\theta, n)$ du dispositif non linéaire étudié est fonction de $\theta$ et de l'indice $n$. Nous avons tracé sur la figure 4 ses variations calculées en fonction de $n$, pour des valeurs de $\theta$ très voisines de $\theta_{0}$. Nous pouvons remarquer que, pour $\theta>\theta_{0}$, si, à faible éclairement $\left(n=n_{0}\right), \mathcal{R}$ est proche du minimum, lorsque la puissance augmente on observera, selon le signe de $\delta$ une augmentation de $\mathcal{R}$ avec passage ou non par la résonance selon que $\delta n$ est positif ou négatif et donc observation ou non de 


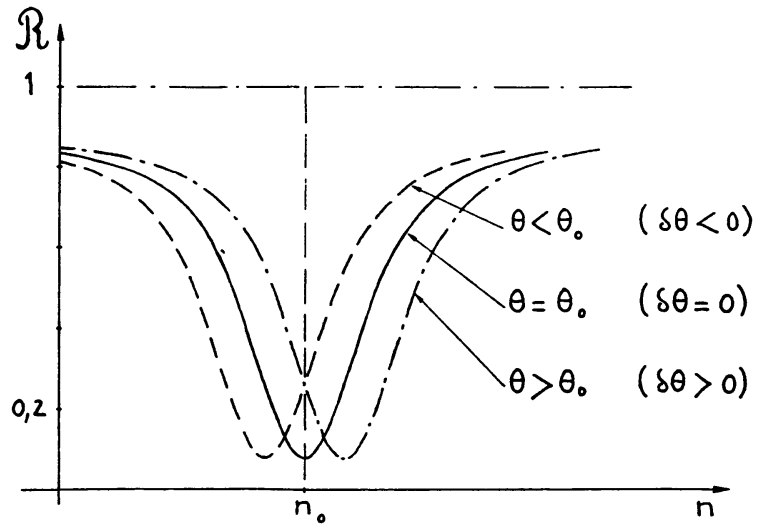

Fig. 4. - Variation du coefficient de réflexion avec l'indice du silicium.

[Reflection coefficient versus the silicon refractive index.]

commutation, le phénomène s'inversant pour $\theta<\theta_{0}$.

La figure 5 montre les résultats obtenus pour différentes valeurs de $\delta \theta=\theta-\theta_{0}$ et différentes valeurs de la puissance incidente crête $\left(P_{\text {ic }}\right)$, les mesures étant effectuées sur les faisceaux incidents (trace 1), dans la région $\mathbf{R}$ (trace 2) et dans la région $\mathrm{G}$ (trace 3). Elle montre, en premier lieu, qu'on n'observe de minimum sur la réflectivité, et de commutation que pour des valeurs positives de $\delta \theta$, ce qui prouve que, dans nos conditions expérimentales, ce sont les effets thermiques qui prédominent.

- Pour $\delta \theta<0$, à faible puissance, la réflectivité varie peu ; quand la puissance augmente, la réflectivité augmente également.

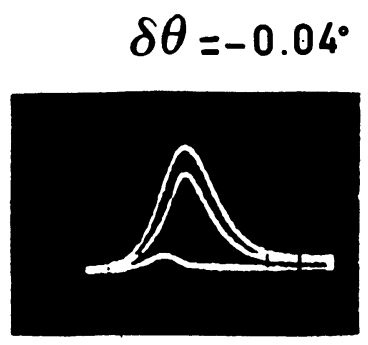

$\mathbf{a}_{1}$

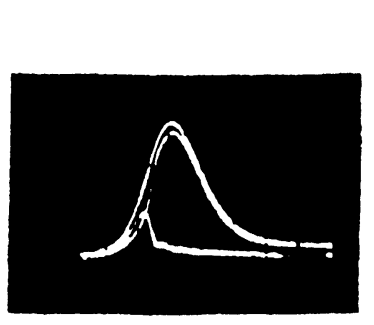

b) $\delta \theta=0^{\circ}$

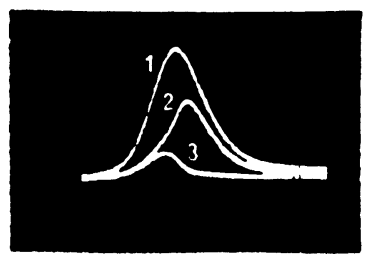

a 2

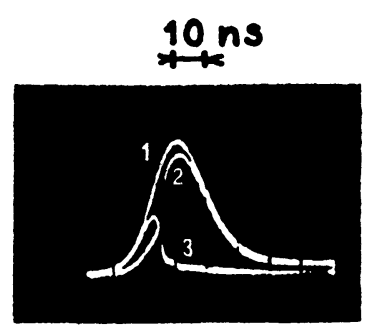

$b_{2}$

- Pour $\delta \theta=0$, à faible puissance, la réflectivité $\mathcal{R}$ est minimale : c'est pour cette valeur de $\theta$ que la croissance du mode guidé est la plus rapide. Quand la puissance incidente $P_{\mathrm{i}}$ augmente, $\mathcal{R}$ augmente également. Pour des valeurs importantes de $\boldsymbol{P}_{\text {ic }}$, on observe des commutations.

- Pour $\delta \theta>0$ la réflectivité décroît pendant que le mode guidé croît, puis $\mathcal{R}$ augmente alors que la puissance guidée décroît. Le passage à la résonance s'observe d'autant plus tardivement que $\delta \theta$ est plus grand. Pour les grandes valeurs de $\boldsymbol{P}_{\text {ic }}$, on observe des commutations. Si nous appelons $\tau_{c}$ la durée d'une commutation, soulignons que pour une valeur fixée de $\delta \theta, \tau_{\mathrm{c}}$ diminue quand $P_{\text {ic }}$ augmente. Pour $\delta \theta=0,11^{\circ}$ et $P_{\text {ic }}=700 \mathrm{~kW} / \mathrm{cm}^{2}$, nous avons observé, à l'aide d'un oscilloscope Thomson TSN660 $(5 \mathrm{GHz})$ et d'une photodiode à avalanche (le temps de montée de l'ensemble étant d'environ $200 \mathrm{ps)}$ un temps $\tau_{\mathrm{c}} \approx 700 \mathrm{ps}$. Pour retrouver une même durée $\tau_{\mathrm{c}}$ quand $\delta \theta$ augmente, il est nécessaire d'augmenter $P_{\text {ic }}$.

Par ailleurs, ces expériences permettent d'observer une commutation mais pas de bistabilité. Cette commutation résultant, au moins en partie, d'une élévation de température, on comprend aisément que le retour à l'état initial ne puisse être observé pendant la durée de l'impulsion (20 ns).

Une étude spatiale des phénomènes a également été effectuée expérimentalement, pour essayer de caractériser l'influence de l'extension finie du faisceau excitateur sur l'évolution du mode guidé et les caractéristiques de la commutation : la figure 6 indique la répartition d'intensité dans le guide, dans la

$$
\delta \theta=0.04^{\circ} \quad \delta \theta=0.11^{\circ}
$$

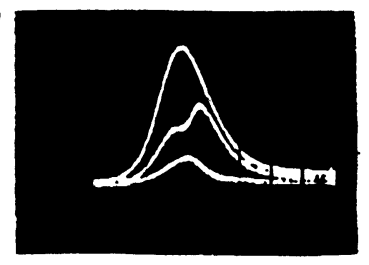

$\mathbf{a}_{3}$

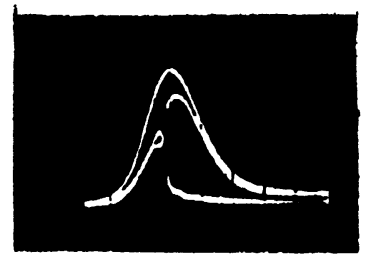

$b_{3}$

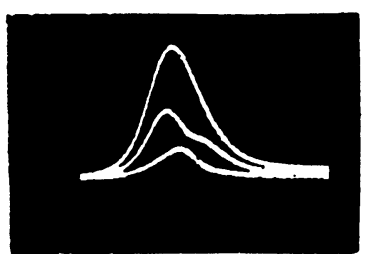

$\mathbf{a}_{4}$

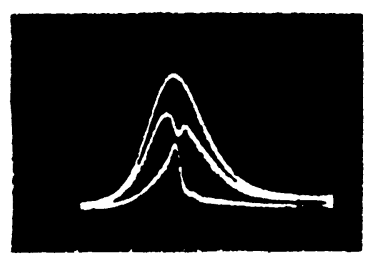

$\mathbf{b}_{4}$

Fig. 5. - Variations temporelles de : 1) l'intensité incidente, 2) l'intensité diffractée dans la région $R, 3)$ l'intensité guidée, pour plusieurs valeurs de $\delta \theta .\left(\mathrm{a}_{\mathrm{i}}: P_{\mathrm{ic}}=135 \mathrm{~kW} / \mathrm{cm}^{2}, \mathrm{~b}_{\mathrm{i}}: P_{\mathrm{ic}}=500 \mathrm{~kW} / \mathrm{cm}^{2}\right.$.)

[Temporal variations of : 1) the incident intensity, 2) the intensity diffracted from the $\mathrm{R}$ region, 3) the guided intensity, for several values of $\delta \theta\left(\mathrm{a}_{\mathrm{i}}: P_{\mathrm{ic}}=135 \mathrm{~kW} / \mathrm{cm}^{2}, \mathrm{~b}_{\mathrm{i}}: P_{\mathrm{ic}}=500 \mathrm{~kW} / \mathrm{cm}^{2}\right)$.] 


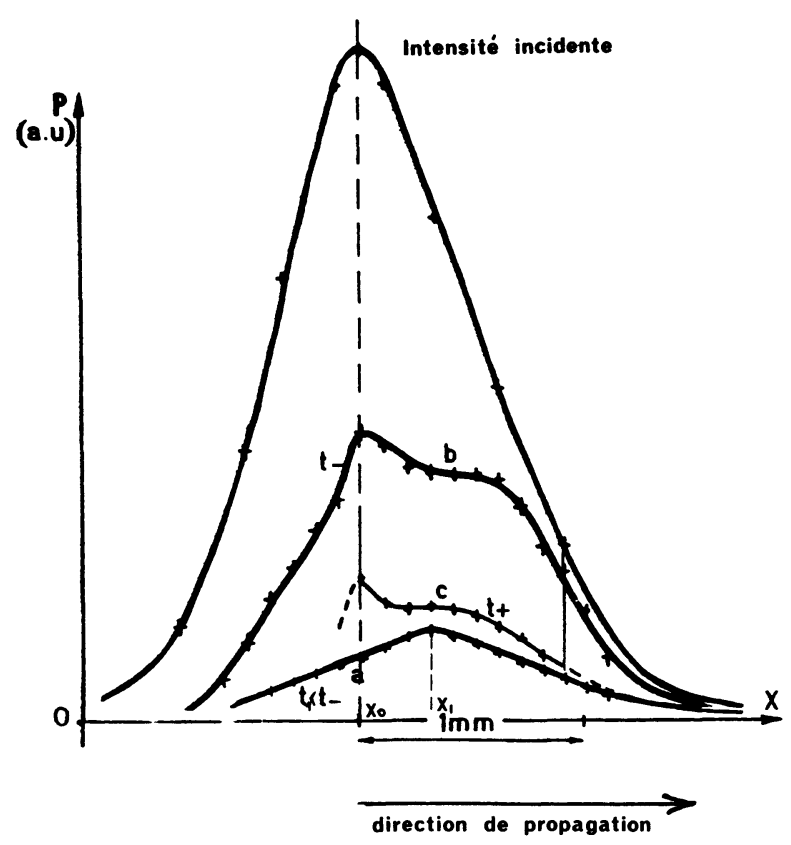

Fig. 6. - Variation spatiale dans la direction de propagation de l'intensité incidente crête, et de l'intensité guidée mesurée à différents instants.

[Spatial dependence of the maximum incident intensity and of the guided intensity, at various times, in the propagation direction.]

direction de propagation, en différents instants. On voit que, au début de l'impulsion excitatrice $\left(t_{1}\right)$, le maximum du mode guidé a lieu en un point $x_{1}$, éloigné de $x_{0}$ (abscisse du maximum d'intensité du faisceau excitateur) : cet écart $x_{1}-x_{0}$ caractérise le couplage entre l'onde incidente et le guide. A des instants ultérieurs $t_{2}\left(t_{1}<t_{2}<t_{-}\right)$, le maximum $x_{2}$ du mode guidé se rapproche de $x_{0}\left(x_{0}<x_{2}<\right.$ $\left.x_{1}\right):$ le couplage augmente. Les courbes $b$ et $c$ donnent la répartition d'intensité dans le guide respectivement aux instants $t_{-}$et $t_{+}$qui précèdent et suivent immédiatement la commutation. A la précision des mesures près, il semble que la commutation ait lieu simultanément dans toute une zone de l'espace dont l'extension dépend de la puissance incidente crête : $\boldsymbol{P}_{\mathrm{ic}}$. Une interprétation théorique plus complète des phénomènes observés nécessiterait une analyse spatio-temporelle du couplage entre un faisceau gaussien et un guide non linéaire. Cette étude est en cours.

\section{Analyse théorique.}

L'interprétation des résultats a été effectuée en faisant les hypothèses simplificatrices suivantes: nous avons supposé une excitation en onde plane et donc négligé les effets transverses. Nous avons également supposé que la désexcitation des paires électron-trou crées par le faisceau excitateur pouvait être décrite phénoménologiquement par un temps de relaxation $\tau$ et que lors de cette désexcitation toute l'énergie absorbée était transformée en chaleur. Dans ces conditions, on peut expliciter les relations donnant la variation d'indice d'origine électronique, proportionnelle au nombre de paires électron-trou $N(t)$ existant à un instant $t$ :

$$
\delta n_{\mathrm{c}}(t)=-K_{\mathrm{e}} N(t) \text { avec } K_{\mathrm{e}} \approx 0,9 \times 10^{-21} \mathrm{~cm}^{3}[5]
$$

et la variation d'indice d'origine thermique

$$
\delta n_{\mathrm{T}}(t)=\frac{\partial n}{\partial T} \delta T(t) .
$$

Nous avons déterminé expérimentalement le coefficient $\partial n / \partial T$, et l'avons trouvé égal à $1,7 \times 10^{-4} \mathrm{~K}^{-1}$, en très bon accord avec celui annoncé par Eichler [6].

3.1 HyPothÈSE DE LA COUCHE ISOLÉE. - Une première série de simulations a été réalisée en supposant que toute la chaleur était absorbée dans le silicium (hypothèse valable si la durée de l'impulsion est faible comparée au temps caractérisant les échanges thermiques avec l'argent ou le saphir). L'élévation de température du silicium s'écrit alors :

$$
\left(\rho C_{\mathrm{p}}\right)_{\mathrm{Si}} \frac{\mathrm{d}(\delta T(t))}{\mathrm{d} T}=\frac{N(t)}{\tau} \hbar \omega
$$

où $\rho$ est la masse volumique du silicium, $C_{\mathrm{p}}$ sa capacité calorifique et $\omega$ la fréquence de l'onde excitatrice. On a donc :

$$
\delta n_{\mathrm{T}}(t)=\frac{\partial n}{\partial T} \cdot \frac{\hbar \omega}{\left(\rho C_{\mathrm{p}}\right)_{\mathrm{Si}} \tau} \int_{0}^{t} N(u) \mathrm{d} u
$$

les relations (1) et (2) montrent que le rapport de $\delta n_{\mathrm{e}}$ à $\delta n_{\mathrm{T}}$ dépend de la forme de $N(t)$; celle-ci est donnée par:

$$
\frac{\mathrm{d} N(t)}{\mathrm{d} t}=-\frac{N(t)}{\tau}+\frac{P_{\mathrm{a}}(t) \cdot \eta}{\hbar \omega}
$$

où $\eta$ est le rendement quantique et $P_{\mathrm{a}}$ la puissance lumineuse absorbée par unité de volume du film, moyennée sur l'épaisseur $e$ du film; elle s'écrit :

$$
P_{\mathrm{a}}(t)=P_{\mathrm{i}}(t) \cdot \frac{\cos \theta}{e} \cdot[1-\mathcal{R}(\theta, n)] .
$$

A partir des équations (1-4), nous voyons que la variation temporelle de l'indice du silicium :

$$
\delta n(t)=\delta n_{\mathrm{e}}+\delta n_{\mathrm{T}}
$$

dépend de 3 paramètres essentiels :

- la forme et la durée de l'impulsion incidente $\left(\right.$ via $\left.P_{\mathrm{i}}(t)\right)$

- la géométrie du dispositif (épaisseur, importance des échanges de chaleur entre le guide et les milieux extérieurs)

- le temps de recombinaison $\tau$ du matériau. 
Nous avons résolu numériquement ce système d'équations couplées. Nous reportons sur la figure 7 le résultat des simulations effectuées pour $\delta \theta=$ $0,04^{\circ}, P_{\text {ic }}=500 \mathrm{~kW} / \mathrm{cm}^{2}$ qu'il faut comparer aux résultats expérimentaux donnés sur la figure 5 $\left(b_{3}\right)$ : le meilleur accord a été obtenu en ajustant le paramètre $\tau$ et en le prenant égal à $100 \mathrm{ps.} \mathrm{Cette}$ valeur est à comparer à celle que nous avons déterminée expérimentalement par des mesures de
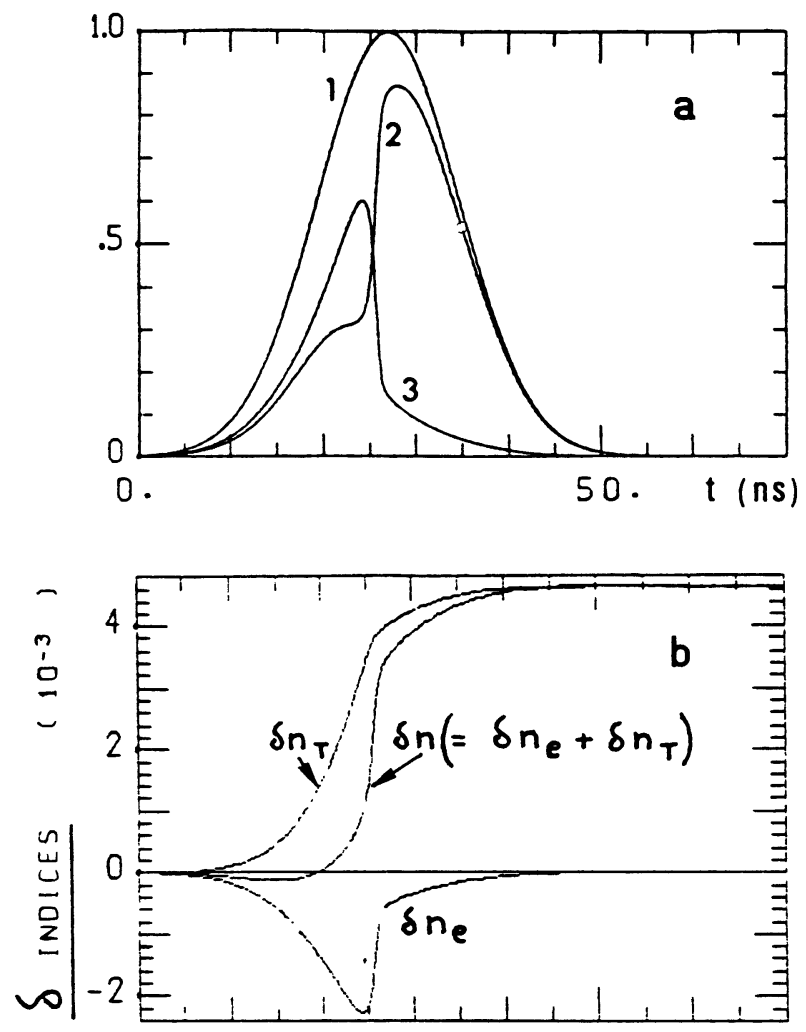

0 .

50. $t(n s)$

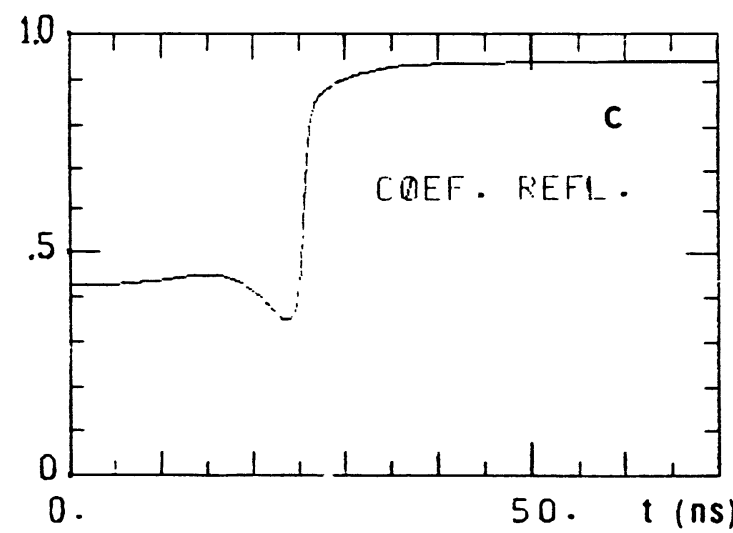

Fig. 7. - Résultats des simulations pour $\delta \theta=0,04^{\circ}$, $P_{\text {ic }}=500 \mathrm{~kW} / \mathrm{cm}^{2}, \Delta t=18 \mathrm{~ns}$ et $\tau=100 \mathrm{ps}$, donnant les variations temporelles : a) des intensités incidente (1), réfléchie (2), et guidée (3), b) des variations d'indice, c) du coefficient de réflexion.

[Simulation results for $\delta \theta=0.04^{\circ}, P_{\text {ic }}=500 \mathrm{~kW} / \mathrm{cm}^{2}$, $\Delta t=18 \mathrm{~ns}$ and $\tau=100 \mathrm{ps}$ : Temporal variation of : a) incident (1), reflected (2) and guided (3) intensities, b) refractive indices, c) reflection coefficient.] photoconduction réalisées sur un échantillon non gravé, non recouvert de métal sur lequel nous avons déposé des électrodes et que nous avons éclairé par des impulsions picosecondes: nous avons alors mesuré une durée de vie des porteurs de 200 ps. Dans ces conditions, les courbes donnant les variations d'indice en fonction du temps expliquent bien les phénomènes observés. En début d'excitation, $\delta n_{\mathrm{c}}$ et $\delta n_{\mathrm{T}}$ ont des valeurs absolues voisines, et des signes opposés : l'indice global reste à peu près constant. Puis, quand les effets thermiques deviennent prédominants, le système passe par la résonance (le coefficient de réflexion par sa valeur minimale) puis en ressort. Le mode guidé se désaccorde alors, provoquant une diminution brutale de $\left|\delta n_{\mathrm{e}}\right|$, qui s'ajoutant à la croissance régulière de $\delta n_{\mathrm{T}}$ provoque une variation globale de l'indice plus rapide encore que celle de $\delta n_{\mathrm{e}}$ et donc une commutation. Par la suite, les effets électroniques étant devenus négligeables, les variations de l'indice sont déterminées par celles de $\delta n_{\mathrm{T}}$.

Dans le modèle choisi, qui néglige les fuites thermiques, on n'observe évidemment pas de retour à l'état initial ; il ne fait pas apparaître non plus de valeur minimale pour la durée $\tau_{\mathrm{c}}$ de la commutation. Ce modèle élémentaire, en onde plane, rend cependant très bien compte des observations expérimentales.

\subsection{INFLUENCE DE LA DIFFUSION DE LA CHALEUR} DANS LE DISPOSITIF. - Nous avons effectué un calcul approché tenant compte de la diffusion de la chaleur dans le substrat de saphir et de la présence d'une couche mince $(2000 \AA)$ métallique déposée sur le silicium. Nous voyons qu'alors, l'élévation maximale de température est deux fois plus faible que celle calculée pour un film isolé et que les variations temporelles de $\delta n_{\mathrm{T}}$ sont modifiées surtout au début de l'impulsion- excitatrice. Ces calculs montrent donc que les effets thermiques réels sont moins importants que ceux précédemment calculés et qu'il est possible, en augmentant l'épaisseur de la couche métallique, de diminuer les effets thermiques, ce qui ne présente pas de difficulté technologique avec la structure en guide plan utilisée.

3.3 INFLUENCE DES AUTRES PARAMÈTRES. - Outre les facteurs géométriques, d'autres paramètres peuvent agir sur la variation temporelle de l'indice du silicium $\delta n(t)$ et donc sur les caractéristiques des commutations et l'existence éventuelle d'une bistabilité : il s'agit, nous l'avons vu, de la durée de l'impulsion excitatrice ou du temps de recombinaison $\tau$.

Pour tenter d'observer un cycle de bistabilité avec des commutations rapides, il est nécessaire de privilégier les effets électroniques. Pour cela nous pouvons raccourcir la durée de l'impulsion incidente, à 
énergie constante. Nous représentons sur la figure 8 les résultats de la simulation effectuée pour une impulsion gaussienne de durée $3 \mathrm{~ns}$, avec $P_{\text {ic }}=$ $3,2 \mathrm{MW} / \mathrm{cm}^{2}, \delta \theta=-0,20^{\circ}$ et $\tau=100 \mathrm{ps}$. Nous voyons (Fig. 8a) que le système passe, au cours de 2 commutations très rapides, d'un état de haute à un état de basse réflectivité, puis retourne à un état de haute réflectivité, l'intensité guidée présentant, simultanément, 2 commutations. En effet, en début d'excitation le système est hors résonance. La variation d'indice d'origine électronique, qui peut très rapidement devenir importante, alors que celle d'origine thermique demeure négligeable, provoque une commutation vers un état de basse réflectivité, état déterminé par la puissance de l'impulsion. Dans cet état, les vitesses de variation de $\delta n_{\mathrm{e}}$ et $\delta n_{\mathrm{T}}$ étant du même ordre, le coefficient de réflexion demeure constant, puis lorsque $\delta n_{\mathrm{T}}$ croît plus vite que $\left|\delta n_{\mathrm{e}}\right|$, le système repasse à la résonance pour en ressortir : on observe une commutation en tout point identique à celle décrite au paragraphe 3.1. Dans l'état final, le système est dans un état de haute réflectivité, différent de l'état initial puisque, les effets thermiques demeurant, $\delta n=\delta n_{\mathrm{T}} \neq 0$.

Nous venons de voir que le dispositif que nous utilisons est susceptible de présenter un fonctionnement bistable s'il est éclairé par des impulsions courtes suffisamment puissantes. Un résultat tout à fait analogue serait obtenu si on diminuait les effets thermiques en augmentant la durée de vie des paires électron-trou.

\section{Conclusion.}

Cette structure en guide d'onde permet donc d'atteindre dans le milieu non linéaire un très grand renforcement du champ et donc des effets non linéaires importants, ceci dans une bande de fréquence relativement large. Dans cette structure, nous avons observé des commutations rapides (quelques centaines de picosecondes), résultant de la compétition entre des effets électroniques et des effets thermiques. Les calculs théoriques nous permettent d'envisager l'observation de commutations encore plus rapides, ne mettant en jeu que des phénomènes électroniques, et de cycles de bistabilité, par réduction des effets thermiques, obtenue en optimisant la thermalisation des dispositifs, en utilisant des impulsions excitatrices plus brèves ou des matériaux ayant des temps de recombinaison plus
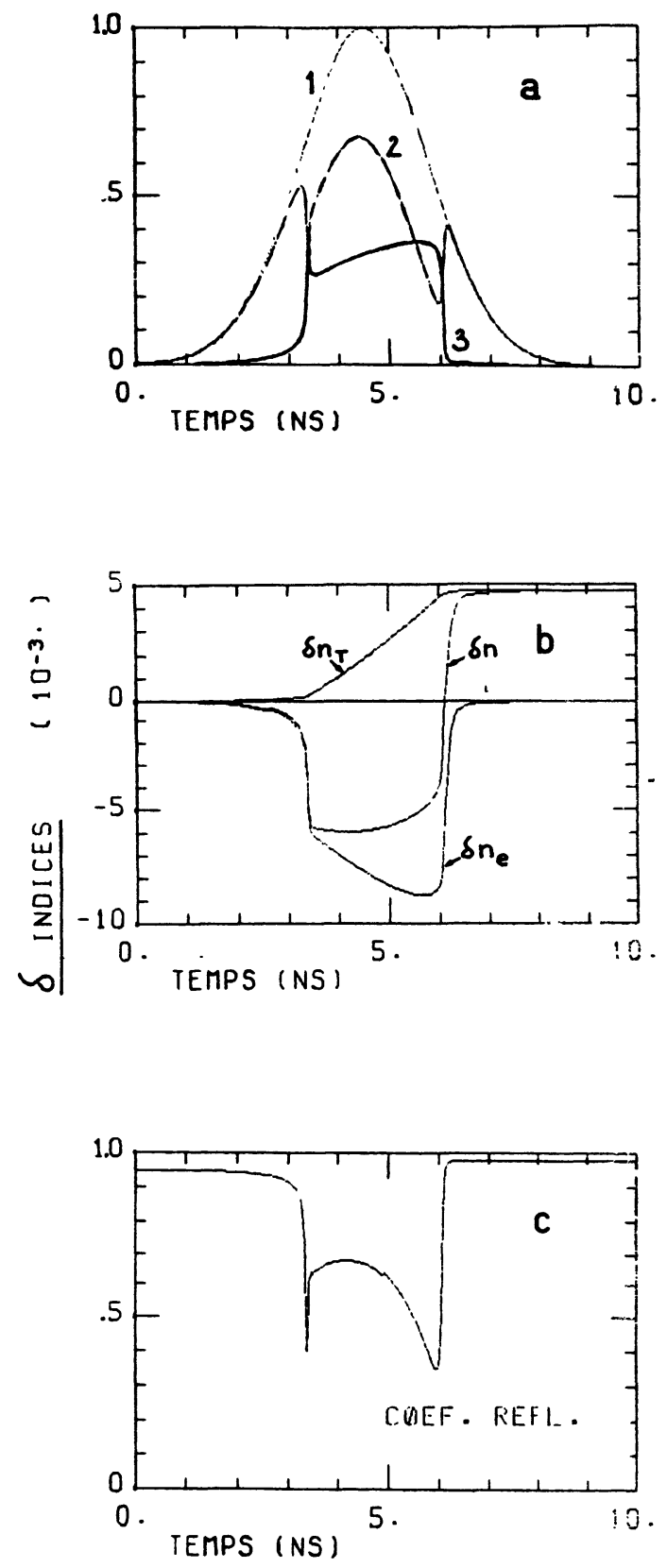

Fig. 8. - Résultats des simulations pour $\delta \theta=-0,20^{\circ}$, $P_{\text {ic }}=3,2 \mathrm{MW} / \mathrm{cm}^{2}, \Delta t=3 \mathrm{~ns}$ et $\tau=100 \mathrm{ps}$.

[Simulation results for $\delta \theta=-0.20^{\circ}, P_{\mathrm{ic}}=3.2 \mathrm{MW} / \mathrm{cm}^{2}$, $\Delta t=3 \mathrm{~ns}$ and $\tau=100 \mathrm{ps}$.]

longs. Cette structure semble donc très compétitive dans le domaine des commutations optiques ultrarapides. 


\section{Bibliographie}

[1] Migus, A., Hulin, D., Mysyrowicz, A., ANtonetti, A., Peyghambarian, N., Gibbs, H. M., Masselink, W. T., Morkoç, H., Optical Bistability III, ed. by H. M. Gibbs, P. Mandel, N. Peyghambarian and S. D. Smith (Springer Verlag Berlin) 1986, p. 42.

[2] Bigot, J. Y., Frindi, M., Wegener, M., HonerLAGE, B., LeVY, R., GRUN, J. B., Optical Bistability III, ed. by H. M. Gibbs, P. Mandel, N. Peyghambarian and S. D. Smith (Springer Verlag, Berlin) 1986, p. 132.
[3] Vincent, P., Paraire, N., Neviere, M., Koster, A., Reinisch, R., J. Opt. Soc. Am. B 2 (1985) 1106.

[4] Garmire, E., Solid State Circuits Conference Proceedings (NEREM) 1972, p. 115.

[5] SpItzer, W. G., FAN, H. Y., Phys. Rev. 106 (1957) 882.

[6] EichleR, H. J., Opt. Commun. 45 (1983) 62. 